\title{
Genetic diversity in a moulting colony of southern elephant seals in comparison with breeding colonies
}

\author{
Wiesław Bogdanowicz ${ }^{1, *, * *}$, Małgorzata Pilot ${ }^{1,3, * *}$, Marta Gajewska $^{1}$, Ewa Suchecka ${ }^{1}$, \\ Mikołaj Golachowski
}

\author{
${ }^{1}$ Museum and Institute of Zoology, Polish Academy of Sciences, Wilcza 64, 00-679 Warszawa, Poland \\ ${ }^{2}$ Department of Antarctic Biology, Institute of Biochemistry and Biophysics, Polish Academy of Sciences, Pawińskiego 5a, \\ 02-106 Warszawa, Poland \\ ${ }^{3}$ Present address: School of Life Sciences, University of Lincoln, Brayford Pool, Lincoln LN6 7TS, UK
}

\begin{abstract}
Southern elephant seals Mirounga leonina migrate seasonally between pelagic foraging areas in the Southern Ocean and breeding and moulting sites on subantarctic islands. Here we characterized genetic diversity of the elephant seal moulting colony from King George Island (KGI), South Shetlands Archipelago, in comparison with breeding colonies described in earlier studies. Although KGI serves as a breeding site, the numbers of elephant seals are up to 3 times higher during the moulting season, suggesting post-breeding immigration from other sites, or that large numbers of individuals skip the breeding season every year. High haplotype diversity and a high percentage of shared haplotypes is consistent with the hypothesis of immigration from other South Atlantic colonies, which is also supported by satellite tracking data from earlier studies. Estimates of effective population size at both mtDNA and microsatellite loci were unexpectedly high compared with the census size of the KGI colony, suggesting that they were elevated due to the presence of immigrants. However, we detected few immigrants and no genetic structure in the KGI colony, which could result from the genetic similarity between KGI and other breeding colonies from the South Atlantic, but could also be an effect of an insufficient sampling scheme. These results show the need for genetic monitoring of southern elephant seal colonies throughout their annual cycle to better understand the range of their seasonal movements and patterns of gene flow. The southern elephant seal serves as a model to study links between spatio-temporal environmental variability, population dynamics, and individual movements, physiology and reproductive behavior. Understanding the patterns of dispersal and gene flow in this species is essential to adequately address these general questions.
\end{abstract}

KEY WORDS: Southern elephant seals · Genetic diversity $\cdot$ Moulting $\cdot$ Mating system $\cdot$ Gene flow $\cdot$ Dispersal · Antarctic

- Resale or republication not permitted without written consent of the publisher

\section{INTRODUCTION}

Southern elephant seals Mirounga leonina are distributed around the Antarctic continent. They spend non-breeding seasons foraging over large distances in the Southern Ocean, except for during moulting periods, when they haul out on beaches (Reeves et al. 2002). During their foraging trips, elephant seals travel hundreds to thousands of kilometers (e.g. Jonker \& Bester 1998, Bradshaw et al. 2004, Tosh et al. 2009). Despite these regular long-distance excursions, they show fidelity to established sites during the annual breeding season (e.g. Hindell \& Little 1988, Lewis et al. 1996, Hofmeyr 2000). The main breeding colonies are located on Macquarie Island (south Pacific Ocean), Kerguelen Islands and Heard 
Island (south Indian Ocean), South Georgia Island (South Atlantic) and Peninsula Valdés (Argentina) (McMahon et al. 2005). A number of smaller breeding colonies also exist on other sub-Antarctic islands. Significant genetic differentiation has been found between the main colonies (Slade et al. 1998, Hoelzel et al. 2001), but some cases of male-mediated genetic dispersal between distant locations have been detected: from Macquarie Island to the Falkland Islands (over 8000 km; Fabiani et al. 2003), and from Marion Island (south Indian Ocean) to Gough Island (South Atlantic, 3860 km; Reisinger \& Bester 2010).

Mirounga leonina is one of the most polygynous mammal species, with high variance in reproductive success among males, as documented by genetic studies (Hoelzel et al. 1999, Fabiani et al. 2004). Observational data suggest a high female to male ratio in harems, varying from 9:1 to 277:1 in different colonies (Carrick et al. 1962, Wilkinson \& van Aarde 1999; this last number may be overestimated as it is based only on behavioural observations). Therefore, successful dispersal (i.e. followed by reproduction) of one male may have a substantial effect on the genetic composition of the entire breeding colony. Male-biased gene flow combined with strong female philopatry suggests weak or moderate population structuring at paternally and bi-parentally inherited markers, and strong structuring at maternally inherited mitochondrial DNA (mtDNA). Indeed, there is a substantial phylogeographic differentiation between the Macquarie Island colony and other colonies (Fabiani et al. 2003, de Bruyn et al. 2009). However, mtDNA haplotypes from the southern Indian Ocean (Marion Island and Heard Island) and the southern Atlantic Ocean (Falklands, Elephant Island and South Georgia) do not form separate haplogroups (de Bruyn et al. 2009). Moreover, the 3 above mentioned colonies from the South Atlantic share common haplotypes (de Bruyn et al. 2009). This suggests either ongoing female-mediated gene flow, or recent common maternal ancestry of these populations.

Although the genetic composition of breeding colonies of elephant seals has been the subject of several studies (e.g. Hoelzel et al. 1993, 2001, Slade et al. 1998, Fabiani et al. 2003, 2004, 2006), the genetic composition of moulting sites has not been yet investigated. Here we studied the elephant seals from King George Island (KGI), South Shetlands Archipelago (South Atlantic). This island serves as a breeding site for elephant seals. However, after the breeding season the numbers of individuals increase up to 3-fold during the moulting season in January (Salwicka \& Rakusa-Suszczewski 2002). This is un- usual in comparison with other breeding sites, including the main South Atlantic breeding colony in South Georgia, where after breeding the numbers of elephant seals decline (e.g. Laws 1984, Slip \& Burton 1999). The aim of our study was to characterize genetic diversity of the elephant seal moulting colony from KGI in comparison with the breeding colonies that have been described in earlier studies. If moulting sites are aggregations of individuals from genetically distinct breeding colonies, it may be expected that genetic diversity of such a site will be higher than in the individual breeding colonies, and that the genetic structure will reflect the presence of individuals of different origin.

\section{MATERIALS AND METHODS}

\section{Fieldwork}

We studied the elephant seal colony from the western shore of Admiralty Bay on KGI during 3 austral summer seasons: 2002/2003, 2003/2004 and 2005/ 2006. The colony is spread over the $15 \mathrm{~km}$ stretch of the shore from Patelnia Point to Point Thomas (Fig. 1C), and occupies 13 ice-free areas separated by rocky cliffs and glaciers (Salwicka \& RakusaSuszczewski 2002). Elephant seals are present in Admiralty Bay throughout the year, and show annual cyclic changes in density and spatial distribution (Salwicka \& Rakusa-Suszczewski 2002). Censuses performed between 1988 and 2000 indicated that on average, $237 \pm 41$ individuals (mean $\pm \mathrm{SE}$ ) were present in the study area in the breeding season in midOctober, and $525 \pm 62$ individuals in the moulting season in mid-January. The number of pups varied from 21 to 314 annually throughout that study period (Salwicka \& Rakusa-Suszczewski 2002).

Most individuals were sampled in the moulting season in January and February, except for 13 pups sampled between the end of November and the end of December. Samples of moulting skin were taken using a comb attached to a long stick (which allowed researchers to maintain distance from sampled individuals). Each sample was placed in a separate Eppendorf tube and kept in the freezer until DNA extraction.

\section{Laboratory methods}

We extracted DNA from skin samples of 275 elephant seals using the Genomic DNA Mini Kit (A \& A Biotechnology). We sequenced $251 \mathrm{bp}$ of the mtDNA 


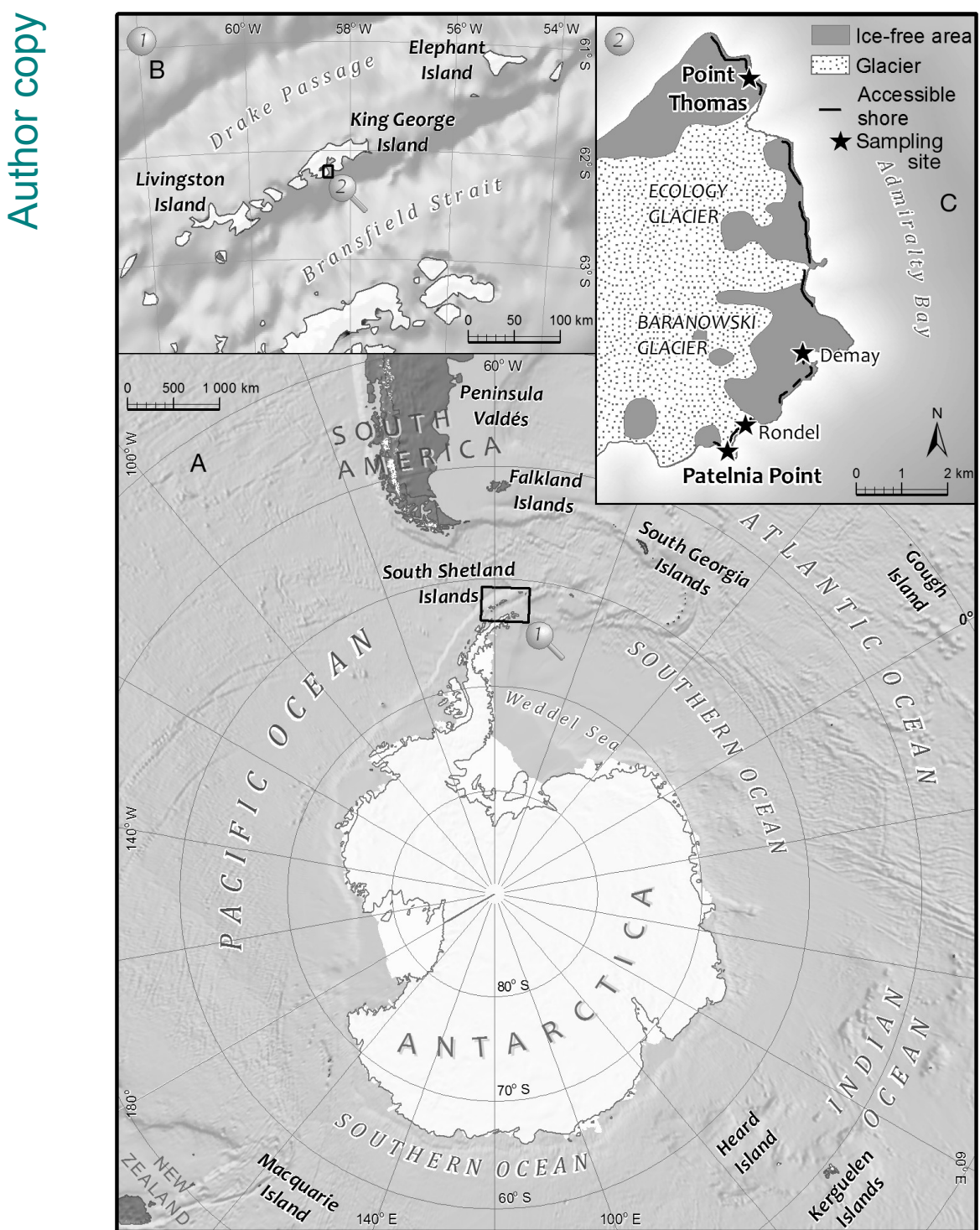

Fig. 1. (A) Subantarctic colonies of southern elephant seals; (B) South Shetlands Archipelago; (C) study area

quencer (Beckman-Coulter). For each individual that had a new and unique haplotype, the sequencing was repeated, and both strands were sequenced to check for the consistency of results.

For 139 individuals, we established genotypes at 12 autosomal microsatellite loci: HI2, HI4, HI8, HI15, HI16, HI20, Lw5, Lw20 (Davis et al. 2002), M2b (Hoelzel et al. 2001), Hg4.2, Hg8.9 and Hg8.10 (Allen et al. 1995). For loci described in Davis et al. (2002), the PCR reaction was carried out in $20 \mu \mathrm{l}$ containing $2 \mu \mathrm{l}$ of template DNA, $2 \mu \mathrm{l}$ of each primer $(2 \mathrm{pmol})$ (with the forward primer fluorescently labelled) and $10 \mu \mathrm{l}$ Multiplex PCR Kit (Qiagen) under the following conditions: $15 \mathrm{~min}$ at $95^{\circ} \mathrm{C}$ followed by 16 cycles of $30 \mathrm{~s}$ at $94^{\circ} \mathrm{C}, 90 \mathrm{~s}$ at $58^{\circ} \mathrm{C}$ and $60 \mathrm{~s}$ at $72^{\circ} \mathrm{C}$, then 10 cycles of $30 \mathrm{~s}$ at $94^{\circ} \mathrm{C}, 90 \mathrm{~s}$ at $57^{\circ} \mathrm{C}$ and $60 \mathrm{~s}$ at $72^{\circ} \mathrm{C}$, and 10 cycles of $30 \mathrm{~s}$ at $94^{\circ} \mathrm{C}, 90 \mathrm{~s}$ at $55^{\circ} \mathrm{C}$ and $60 \mathrm{~s}$ at $72^{\circ} \mathrm{C}$, followed by $30 \mathrm{~min}$ at $60^{\circ} \mathrm{C}$. For the remaining loci (M2b, Hg4.2, Hg8.9 and Hg8.10) each PCR mix (volume $15 \mu \mathrm{l}$ ) contained $1.5 \mu \mathrm{l}$ genomic DNA, $0.5 \mu \mathrm{l}$ labelled forward primer $(5 \mathrm{pmol}), 0.5 \mu \mathrm{l}$ reverse primer (5 pmol) and $7.5 \mu \mathrm{l}$ REDTaq ReadyMix (Sigma-Aldrich). The PCR profile used was: $3 \mathrm{~min}$ at $96^{\circ} \mathrm{C}$ followed by 35 cycles of $30 \mathrm{~s}$ at $96^{\circ} \mathrm{C}, 45 \mathrm{~s}$ at $55^{\circ} \mathrm{C}, 45 \mathrm{~s}$ at $72^{\circ} \mathrm{C}$, and a final elongation for $5 \mathrm{~min}$ at $72^{\circ} \mathrm{C}$. Allele lengths were determined using a $\mathrm{CEQ}^{\mathrm{TM}} 8000$ sequencer (Beckmann-Coulter).

For some of the sampled individuals, mostly pups, the sex was unknown or

control region for all these individuals, using the primers MLd3 5'-CCA CCA TCA GCA CCC AAA G3' and MLd4 5'-CGG AGC GAG GAT TAG GTA CA$3^{\prime}$. PCR amplification was carried out in a total volume of $50 \mu$ containing $5 \mu \mathrm{l}$ template DNA, $1 \mu \mathrm{l}$ of each primer (10 pmol) and $25 \mu$ of PCR REDTaq ReadyMix PCR Reaction Mix with $\mathrm{MgCl}_{2}$ (SigmaAldrich) using the following thermal profile: $2 \mathrm{~min}$ at $95^{\circ} \mathrm{C}$ followed by 35 cycles of $15 \mathrm{~s}$ at $94^{\circ} \mathrm{C}, 20 \mathrm{~s}$ at $55^{\circ} \mathrm{C}, 1 \mathrm{~min}$ at $72^{\circ} \mathrm{C}$, and a final elongation for $3 \mathrm{~min}$ at $72^{\circ} \mathrm{C}$. Amplified products were purified using the CleanUp PCR Purification Kit (A \& A Biotechnology). Sequencing was carried out in a CEQTM8000 seuncertain, and therefore we used molecular sexing. At first, we used the primers ZFYF, ZFYR, ZFXF and ZF2RA, described by Curtis et al. (2007), to amplify fragments of the ZFX and ZFY genes for a few goodquality samples. Because of degraded DNA matrices, we were unable to amplify long DNA fragments (931-1045 bp) produced by these primers for the remaining samples. Therefore, we designed primers targeting smaller $(249 \mathrm{bp}$ ) fragments of the ZFX and ZFY genes: MirXF 5'-GCA TTT TAG CTG CTA GAC CA-3' and MirXR 5'-AAC ATA ATC CAA TTC TTT GC-3', and MirYF 5'-GTT AAC GAT AGC AAT TTA GCT G-3' and MirYR 5'-AAT TCC TGC TTC ATA 
CTT ACA-3'. PCR amplification was performed in a total volume of $20 \mu \mathrm{l}$ containing $10 \mu \mathrm{l}$ REDTaq ReadyMix (Sigma-Aldrich), $0.7 \mu \mathrm{l}$ of each primer and $5 \mu$ of template DNA. The PCR thermal profile was as follows: $3 \mathrm{~min}$ at $96^{\circ} \mathrm{C}$ followed by 35 cycles of $30 \mathrm{~s}$ at $96^{\circ} \mathrm{C}, 45 \mathrm{~s}$ at $55^{\circ} \mathrm{C}, 45 \mathrm{~s}$ at $72^{\circ} \mathrm{C}$, and a final elongation for $5 \mathrm{~min}$ at $72^{\circ} \mathrm{C}$. The PCR products were visualized on a $1.5 \%$ agarose gel.

\section{Analysis of genetic variability and population differentiation}

mtDNA sequences were aligned using the CLUSTALW algorithm implemented in MEGA 5.05 (Tamura et al. 2011) to identify distinct haplotypes. Haplotype and nucleotide diversity in the KGI colony were calculated in ARLEQUIN 3.5 (Excoffier \& Lischer 2010). We compared haplotypes found in the KGI moulting colony with haplotypes from other southern elephant seal colonies that were obtained from Hoelzel et al. (1993), Slade et al. (1998), Fabiani et al. (2003) and GenBank (DQ267952, DQ267961). Phylogenetic relationships between haplotypes were reconstructed using the median-joining network approach implemented in the software NETWORK 4.510 (Bandelt et al. 1999). To assess whether our sampling effort accurately reflects the overall haplotype variability in the KGI colony, we used the program SPIDER (Brown et al. 2012) to construct a rarefaction curve showing how the number of haplotypes increases with the increasing sampling effort. Because the order in which the subsequent sequences are added affects the shape of the curve, we performed 1000 permutations that simulated the accumulation of haplotypes in a random order. We also used the same program to calculate the Chao1 estimate of the total number of haplotypes in a population based on the number of haplotypes sampled, and the number of singletons and doubletons in the data set (Chao \& Lee 1992). For a comparison, we also evaluated sampling effort in 2 other colonies, Peninsula Valdes and South Georgia, using the same methods.

The microsatellite data set for the KGI colony was checked for the presence of genotyping errors in MICROCHECKER (van Oosterhout et al. 2004). We used GENALEX (Peakall \& Smouse 2006) to calculate measures of genetic variability at microsatellite loci: number of alleles, observed $\left(H_{\mathrm{O}}\right)$ and expected heterozygosity $\left(H_{\mathrm{E}}\right)$, and the inbreeding coefficient $\left(F_{\text {IS }}\right)$. Deviations from Hardy-Weinberg equilibrium and their direction (heterozygote deficit or excess) were assessed using the exact test implemented in
GENEPOP (Raymond \& Rousset 1995), with 10000 dememorization steps followed by 1000 batches with 10000 Markov chain Monte Carlo (MCMC) iterations per batch.

Genetic structuring was examined using STRUCTURE 2.2 software (Pritchard et al. 2000). We assumed a model of population admixture and correlated allele frequencies among groups, and provided no prior information on population structure. The analysis was run for $100000 \mathrm{MCMC}$ iterations preceded by a burn-in period of 10000 iterations, for the number of groups $(K)$ ranging from 1 to 5 , and was repeated 3 times for each $K$ to check the consistency of results. For each $K$, we checked whether the run parameters (likelihood, posterior probability of data and alpha) reached convergence within the burn-in period. Population differentiation was also assessed with principal coordinates analysis (PCA) based on pairwise genetic distances between individuals using GENALEX.

We also specifically tested for genetic differentiation between groups of individuals sampled in 3 different moulting seasons. For this purpose, we calculated pair-wise $F_{\mathrm{ST}}$ values between these groups, and tested for significance by performing 10000 permutations. We also performed the exact test of population differentiation (Raymond \& Rousset 1995), with 100000 Markov chain steps and 10000 dememorization steps. Additionally, we performed analysis of molecular variance (AMOVA) ( Excoffier et al. 1992), with 1000 permutations. These analyses were performed in ARLEQUIN 3.5. We did not test for spatial genetic differentiation, because the study area (15 km stretch of the shore) was too small as compared with the migratory potential of this species (thousands kilometers) to allow for any meaningful interpretation of such results.

\section{Demographic estimates}

We estimated the effective size $\left(N_{\mathrm{e}}\right)$ of the population in each year from microsatellite allele frequency data using a point estimation method based on linkage disequilibrium, and a moment-based temporal method, both implemented in NEESTIMATOR 1.3 (Ovenden et al. 2007). This analysis was performed for each moulting season separately, as well as for all the samples pooled together. Wherever there was a sufficient number of individuals (i.e. for the season 2005/2006 and for the entire data set), we partitioned individuals into 2 cohorts: pups and adults. More precise subdivision was impossible because we had no 
data on the age of adult individuals. We excluded 2 individuals identified as immigrants (see below) from this analysis.

We also estimated female effective population size from nucleotide diversity of mtDNA control region sequences. For this analysis, we used the substitution rate of $9.8 \times 10^{-7}$ estimated for the southern elephant seals based on ancient DNA data by de Bruyn et al. (2009). We assumed a generation time of $8 \mathrm{yr}$, which was estimated by Slade et al. (1998) based on female life tables of the stable population at South Georgia (McCann 1985).

To test whether the study population shows signs of a genetic bottleneck in the period of last few generations, we applied Cornuet \& Luikart's (1996) tests implemented in the program BOTTLENECK 1.2.02 (Piry et al. 1999) for the microsatellite data. We assumed either the strict stepwise mutation model (SMM) or the 2-phase mutation model (TPM) with $95 \%$ single-step mutations.

We also performed a number of tests for population expansion based on the mtDNA data, using ARLEQUIN 3.5. We analysed the mismatch distribution, i.e. the distribution of the observed number of differences between pairs of haplotypes. For populations that passed through a recent demographic expansion (Rogers 1995) or though a range expansion with high levels of migration between neighboring demes (Excoffier 2004), unimodal mismatch distribution is expected. The confidence intervals for the observed distribution were estimated with 1000 bootstrap replicates. We also performed Fu's $F_{S}$ (Fu 1997) and Tajima's $D$ (Tajima 1989) tests of selective neutrality, where significant negative values indicate a demographic expansion.

\section{Detection of immigrants and sex-biased dispersal}

To identify first-generation immigrants we used GENECLASS2 (Piry et al. 2004). Using the frequency method of Paetkau et al. (1995), we calculated the log-likelihood that an individual belongs to the study population from a given year, and then we assessed the probability of an individual being a resident using the Monte Carlo resampling algorithm of Paetkau et al. (2004), with 10000 simulated individuals and a type I error of 0.01 . Because we did not have the genetic data for the potential source populations, immigrants were identified as genetic outliers from the population studied rather than based on their genetic similarity to another population. This may lead to a high level of type II error (unidentified immigrants), but it does not affect type I error. Thus, this method may be expected to underestimate the number of immigrants.

GENALEX (Peakall \& Smouse 2006) was used to calculate the assignment index (AIc) for each individual, and test for sex-biased dispersal by comparing mean AIc values between males and females.

\section{Parentage and kinship analysis}

Because we studied the moulting colony, we did not have the data that would allow us to perform a parentage reconstruction, such as a priori information about mother-offspring pairs that was available in studies of breeding colonies of this species (e.g. Fabiani et al. 2004). Here we used parentage analysis to obtain information about general kinship patterns rather than to study the seals' mating system. For example, detection of a large number of parent-offspring pairs in this moulting colony would suggest a moulting site fidelity of southern elephant seals. In this way, the kinship-based approach may be complementary to population-based genetic inference (see Palsbøll et al. 2010).

Parentage analysis was performed using a maximum-likelihood approach implemented in CERVUS 3.0 (Marshall et al. 1998, Kalinowski et al. 2007), and details of this analysis are described in the Supplement (see www.int-res.com/articles/suppl/m478p287_ supp.pdf). CERVUS was also used to test for the presence of null alleles in the microsatellite data set and estimate their frequencies, for a comparison with MICROCHECKER results. We also applied the program KINGROUP (Konovalov et al. 2004), which implements a maximum-likelihood approach for reconstructing groups of kin at a given level of relatedness. We tested relationships at the full-siblings level (which corresponds to a relatedness level of $R \geq 0.5$, and also includes parent-offspring pairs) and the halfsiblings level (which corresponds to $\mathrm{R} \geq 0.25$, and includes full-siblings, half-siblings, parents and grandparents). We compared KINGROUP and CERVUS results to test for the consistency of kinship inference.

\section{RESULTS}

\section{Genetic diversity and population differentiation}

We detected 58 mtDNA haplotypes among the analysed samples (Table S1 in the supplement), and 
found high haplotype and nucleotide diversity (Table 1). Twenty-five (43\%) haplotypes were unique for KGI (GenBank accession numbers: JX847037JX847061), and 31 haplotypes have been found earlier for other South Atlantic islands: 14 haplotypes for the Falklands (out of 20 detected there; Fabiani et al. 2003), 12 for Elephant Island (which were all the haplotypes detected there; Fabiani et al. 2003) and 5 for South Georgia (out of 28 detected there; Hoelzel et al. 1993, Slade et al. 1998). Additionally, 2 haplotypes from KGI have been found earlier on Marion Island, South Indian Ocean (GenBank DQ267952, DQ267961), one of which also occurred on Heard Island (Slade et al. 1998). Haplotypes unique for KGI were detected in $20 \%$ of individuals, while $79 \%$ of individuals had haplotypes shared with other South Atlantic islands, and only $1 \%$ of individuals had haplotypes shared with the South Indian Ocean islands. We found no common haplotypes between

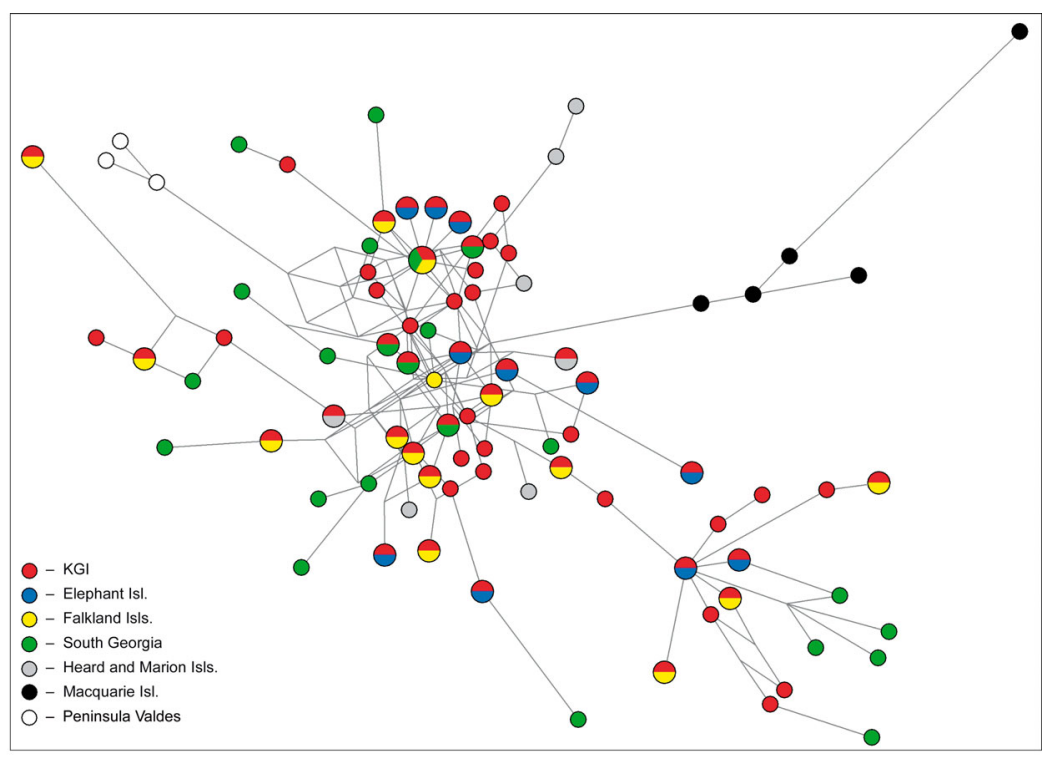

Fig. 2. Mirounga leonina. Median joining network representing phylogenetic relationships between mtDNA haplotypes of southern elephant seals from the King George Island (KGI) moulting colony and other colonies within the range of this species. The size of the circles does not represent the relative frequency of a given haplotype, but reflects the fact of haplotype sharing between different colonies. Haplotype data were obtained from Hoelzel et al. (1993), Slade et al. (1998), Fabiani et al. (2003), GenBank (DQ267952, DQ267961) and the present study
KGI and Macquarie Island (Fig. 2).

The rarefaction curve for KGI showed that the sampling effort was not sufficient to collect all the haplotypes (Fig. S1 in the supplement at www.intres.com/articles/suppl/m478p287_supp.pdf), and the Chao1 estimate of the total number of haplotypes was 109, suggesting that approximately $53 \%$ of haplotypes were sampled. For South Georgia, the rarefaction curve showed almost a linear relationship between the numbers of individuals sampled and haplotypes found (Fig. S1), and the Chao1 estimate was 266 , suggesting that only $9 \%$ of haplotypes were sampled. In contrast, the rarefaction curve for Penin- sula Valdés showed that the sampling effort was sufficient to collect all the haplotypes, and the Chao1 estimate was 3, consistent with the number of haplotypes detected.

The genetic diversity at microsatellite loci in the KGI colony was comparable with other southern elephant seal colonies (Table 2): the mean ( \pm SE) number of alleles per locus was $6.92 \pm 0.93, H_{\mathrm{O}}$ was $0.552 \pm 0.072, H_{\mathrm{E}}$ was $0.588 \pm 0.068$ and $F_{\mathrm{IS}}$ was $0.092 \pm 0.042$. All these variables (number of alleles, $H_{\mathrm{O}}, H_{\mathrm{E}}$ and $\left.F_{\mathrm{IS}}\right)$ were higher in adult males as compared with adult females and pups, but the differ-

Table 1. Mirounga leonina. Genetic diversity at mtDNA control regions and microsatellite loci in different groups of individuals from the King George Island elephant seal population. $N_{\text {hap }}$ : number of haplotypes; $N_{\mathrm{a}}$ : mean number of alleles at microsatellite loci; $H_{\mathrm{O}}$ and $H_{\mathrm{E}}$ : expected and observed heterozygosity, respectively; $F_{\mathrm{IS}}$ : inbreeding coefficient. Standard error for each estimate is shown in parentheses. Sample sizes used in mtDNA and microsatellite analyses, respectively, are shown in parentheses for each demographic group

\begin{tabular}{|c|c|c|c|c|c|c|c|}
\hline \multirow{2}{*}{$\begin{array}{l}\text { Demographic } \\
\text { group }\end{array}$} & \multirow{2}{*}{$\overline{N_{\text {hap }}}$} & \multicolumn{2}{|c|}{ - mtDNA } & \multirow[b]{2}{*}{$N_{\mathrm{a}}$} & \multicolumn{2}{|c|}{-Microsatellite loci } & \multirow[b]{2}{*}{$F_{\mathrm{IS}}$} \\
\hline & & $\begin{array}{l}\text { Haplotype } \\
\text { diversity }\end{array}$ & $\begin{array}{c}\text { Nucleotide } \\
\text { diversity }\end{array}$ & & $H_{\mathrm{O}}$ & $H_{\mathrm{E}}$ & \\
\hline All individuals $(275,139)$ & 58 & $0.957(0.005)$ & $0.022(0.012)$ & $6.917(0.925)$ & $0.553(0.072)$ & $0.589(0.068)$ & $0.092(0.042)$ \\
\hline Adult males $(53,53)$ & 26 & $0.951(0.013)$ & $0.027(0.014)$ & $6.250(0.922)$ & $0.570(0.072)$ & $0.601(0.065)$ & $0.084(0.051)$ \\
\hline Adult females $(45,45)$ & 24 & $0.953(0.017)$ & $0.025(0.014)$ & $5.917(0.839)$ & $0.541(0.070)$ & 0.575 (0.069) & $0.055(0.047)$ \\
\hline Pups $(41,41)$ & 21 & $0.948(0.017)$ & $0.026(0.014)$ & $5.583(0.830)$ & $0.533(0.080)$ & $0.565(0.071)$ & $0.061(0.054)$ \\
\hline
\end{tabular}


ences were non-significant (Table 1). We detected significant deviations from Hardy-Weinberg equilibrium towards a heterozygosity deficit $(\mathrm{p}<0.001)$. A heterozygosity deficit and a high $F_{\mathrm{IS}}$ coefficient may indicate inbreeding, but may also result from the Wahlund effect, sampling bias or genotyping errors such as allelic dropout or the presence of null alleles. It is likely that a sampling bias played an important role here, as the $F_{\mathrm{IS}}$ estimate was significantly positive only in one of the 3 moulting seasons (Table S2 in the supplement).

Null alleles are likely to be encountered in populations with large effective sizes, and those that have diverged from the population that served as a source for the primer design (Chapuis \& Estoup 2007), and this was the case for the study population. MICROCHECKER detected no allelic dropout, but null alleles were detected in 5 loci, with frequencies ranging from 0.06 to 0.12 . However, these frequencies were highly inconsistent between the 4 methods of their estimation (SD ranging from 0.02 to 0.10 ). Moreover, an alternative method of null allele estimation implemented in CERVUS indicated null alleles in only 2 loci. We considered the advantages and disadvantages of correcting the data for null alleles, and decided to perform further analyses on uncorrected data. This was justified by the ambiguity of null allele estimation between the methods, and the fact that these methods are based on the assumption of Hardy-Weinberg equilibrium. This is not necessarily true for our population, because we observed deviations from the equilibrium also in loci that showed no signs of null alleles or other genotyping errors. Moreover, the main problem with the presence of null alle- les is that they lead to overestimation of $F_{\mathrm{ST}}$ and genetic distances (Chapuis \& Estoup 2007), but we detected no structure in our population (see below).

Another possible cause of heterozygosity deficit could be the Wahlund effect, which would occur if the moulting colony were composed of 2 or more genetically distinct groups (e.g. residents and immigrants from other breeding colonies). However, we did not detect any population genetic structure in the analysed sample set. STRUCTURE analysis showed the highest posterior probability at $K=1$, and for higher $K$ values the assignment of individuals to each of the inferred groups was close to equal (a pattern typical when there is no population structure). Consistently, the PCA did not reveal clustering of individuals that would suggest population differentiation.

Pair-wise $F_{\mathrm{ST}}$ values between groups of individuals sampled in 3 different moulting seasons were $-0.0004,0.0047$ and 0.0083 , and only the last value was significant $(\mathrm{p}=0.042)$. This significant $F_{\mathrm{ST}}$ value was between groups most distant in time, which suggests that a temporal equivalent of isolation by distance may occur in the KGI population. However, the exact test of population differentiation did not show any significant differences among the 3 temporal groups, neither pair-wise nor globally $(\mathrm{p}=1.00$ in each case). AMOVA showed that most of the genetic variation was explained by differentiation within individuals $(96.75 \%)$, and differentiation among individuals within temporal groups $(2.92 \%)$. Differentiation among temporal groups explained only $0.33 \%$ of the total variation, and was non-significant $(p=0.84)$. These results further support the lack of genetic structure within the study population.

Table 2. Mirounga leonina. Comparison of genetic diversity at mtDNA control regions ( $N_{\text {hap }}$ and nucleotide diversity) and microsatellite loci $\left(H_{\mathrm{E}}\right.$ and $H_{\mathrm{O}}$ ) (see Table 1) between King George Island and other elephant seal colonies. Other colonies are breeding colonies sampled in the breeding season, except for the extinct population from the Victoria Land Coast that could have been both a breeding colony and a moulting site, and where genetic diversity was assessed based on the study of ancient DNA (de Bruyn et al. 2009). Sample sizes reported with haplotype numbers concern mtDNA analyses only and may be different for microsatellite analyses

\begin{tabular}{|c|c|c|c|c|c|}
\hline \multirow[t]{2}{*}{ Population } & \multicolumn{2}{|c|}{$\longrightarrow$ mtDNA -} & \multicolumn{2}{|c|}{ Microsatellite loci } & \multirow[t]{2}{*}{ Source } \\
\hline & $\begin{array}{c}N_{\text {hap }} \\
\text { (sample size) }\end{array}$ & $\begin{array}{c}\text { Nucleotide } \\
\text { diversity }\end{array}$ & $H_{\mathrm{E}}$ & $H_{\mathrm{O}}$ & \\
\hline King George Island & $58(275)$ & 0.022 & 0.589 & 0.553 & Present study \\
\hline South Georgia & $24(28)$ & 0.028 & 0.590 & 0.624 & Fabiani (2002), Hoelzel et al. (2001) \\
\hline Peninsula Valdez & $3(32)$ & 0.003 & 0.588 & 0.575 & Fabiani (2002), Hoelzel et al. (2001) \\
\hline Falklands & $20(57)$ & 0.032 & 0.655 & 0.656 & Fabiani (2002), Fabiani et al. (2004) \\
\hline Elephant Island & $12(30)$ & 0.032 & - & - & Fabiani (2002) \\
\hline Heard Island & $6(6)$ & 0.023 & - & - & Fabiani (2002) \\
\hline Macquarie Island & $16(49)$ & 0.020 & - & - & de Bruyn et al. (2009) \\
\hline $\begin{array}{l}\text { Victoria Land coast } \\
\text { (ancient population) }\end{array}$ & $177(223)$ & $0.024 / 0.022^{\mathrm{a}}$ & - & - & de Bruyn et al. (2009) \\
\hline
\end{tabular}




\section{Demographic estimates}

Effective population size estimated using the linkage disequilibrium method varied greatly between different years of the study, from 65 to 501 individuals (Table 3). For the pooled data set consisting of individuals collected in each year, effective size was estimated at 771 individuals using the linkage disequilibrium (LD) method and 302 using the moments-based temporal method (Table 3). Some of these estimates had very wide confidence intervals (Table 3). The female effective population size estimated from nucleotide diversity for the entire data set was 3571 individuals.

We found no evidence of a genetic bottleneck within the last few generations (where 10 generations correspond to approximately $80 \mathrm{yr}$ ). For both the SMM and the TPM, we found a significant deviation from the mutation-drift equilibrium, but towards heterozygosity deficit rather than excess (Wilcoxon's signed-ranks one-tailed test for heterozygote deficit, $p=0.001$ for SMM and $p=0.007$ for TPM). The mode-shift test (a descriptor of the allele frequency distribution that discriminates between bottlenecked and stable populations) was non-significant for both mutation models.

The observed mismatch distribution for mtDNA haplotypes was consistent with the expected distribution for the demographic expansion model as well as the spatial expansion model (Fig. S2 in the supplement). For the demographic expansion model, the sum of square deviations (SSD) index and the raggedness index (RI) were low $(\mathrm{SSD}=0.0020, \mathrm{RI}=0.0075)$, but non-significant. For the spatial expansion model, both these indices were low $(\mathrm{SSD}=0.0037, \mathrm{RI}=$ $0.0075)$, but only RI was significant $(\mathrm{p}=0.02)$. Tajima's $D$ test was non-significant $(0.47, \mathrm{p}=0.77)$, while Fu's $F_{S}$ test was significant $(-19.28, \mathrm{p}=0.003)$.

Table 3. Mirounga leonina. Estimates of effective population size in the King George Island elephant seal population based on microsatellite data, using the linkage disequilibrium (LD) and moments-based temporal methods. -: analysis could not be performed for this data subset

\begin{tabular}{|lcccc|}
\hline Year & Cohort & $\begin{array}{c}\text { Sample } \\
\text { size }\end{array}$ & $\begin{array}{c}\text { LD method } \\
(95 \% \text { CI })\end{array}$ & $\begin{array}{c}\text { Temporal method } \\
(95 \% \text { CI })\end{array}$ \\
\hline 2003 & All ind. & 28 & $65(40-147)$ & - \\
2004 & All ind. & 35 & $95(58-218)$ & - \\
2005 & All ind. & 74 & $501(216-\infty)$ & $87(15-\infty)$ \\
2005 & Adults & 63 & $152(99-293)$ & - \\
2005 & Pups & 11 & $57(24-\infty)$ & - \\
All years & All ind. & 137 & $771(385-11,087)$ & $302(49-\infty)$ \\
All years & Adults & 96 & $197(139-321)$ & - \\
All years & Pups & 41 & $283(112-\infty)$ & - \\
\hline
\end{tabular}

\section{Immigration and sex-biased dispersal}

Based on the GENECLASS2 analysis, we detected only one immigrant at the $\mathrm{p}<0.01$ threshold (named here M1, $\mathrm{p}<0.00001)$. One additional immigrant was detected at the $\mathrm{p}<0.05$ threshold (named here M2, $\mathrm{p}=0.013$ ). Both these individuals were adult males. M1 had a unique haplotype KGI43 that has not been found in any other individual from KGI or other islands. M2 had a haplotype KGI11 that was unique for KGI but occurred in 5 other individuals there.

The mean values of the assignment index AIc calculated in GENALEX were positive in females (0.284, SD 0.269) and negative in males $(-0.200, \mathrm{SD}$ 0.287 ), indicating that males are the more dispersing sex. However, differences between the mean AIc values of males and females were not statistically significant ( $U$-test, $Z=0.936, \mathrm{p}=0.175$ ). The most negative AIc value was assigned to M1, giving further support for his immigrant status.

\section{Parentage and kinship analysis}

CERVUS analysis indicated an unusually high number of pairs of individuals with no mismatching loci in the maternity or paternity assignment (see supplement for details), which could result from low genetic variability of southern elephant seals at microsatellite loci. CERVUS assigned 2 putative mother-offspring pairs at a strict confidence level $(95 \%)$ and 22 pairs at a relaxed confidence level $(80 \%)$. Only 2 of these 24 pairs (one assigned with a strict and one with a relaxed confidence level) had matching mtDNA haplotypes and age class (adult/ pup) consistent with the mother- offspring relationship. Individuals from the remaining pairs had different mtDNA haplotypes, inconsistent with a motheroffspring or a full-sibling relationship (mismatching haplotypes were double-checked, see supplement). These pairs of individuals could be more distant relatives, e.g. paternal half-siblings (see Discussion). However, we cannot exclude the possibility that these pairs were matched by chance due to the limited number of loci and low genetic variability.

CERVUS also assigned 4 putative father-offspring pairs at a strict confidence level and 11 pairs at a relaxed confidence level. Nine of these 15 pairs (3 assigned with a strict and 6 with a relaxed confidence) were consistent with the father-off- 
spring relationship based on individuals' age class. Individuals from the remaining 6 pairs had different mtDNA haplotypes, inconsistent with a full-sibling relationship. These pairs could be paternal half-siblings or other more distant relatives, or could have been matched by chance.

KINGROUP analysis indicated 41 groups, consisting of 2 to 4 individuals, as being related at a fullsibling level. Among these groups there were 7 putative father-offspring pairs and 7 putative motheroffspring pairs identified in CERVUS. In only 2 groups identified in KINGROUP did individuals have the same mtDNA haplotypes, so only these 2 groups could consist of full siblings or mother-offspring pairs. The remaining groups could represent paternal half-siblings or father-offspring pairs. However, similar to the case of CERVUS, we cannot exclude the possibility that some of these groups were matched by chance due to the limited number of loci and relatively low genetic variability.

KINGROUP also identified 33 groups, consisting of 2 to 8 individuals, as being related at a half-sibling level. Only 9 of 139 analysed individuals did not belong to any of these groups; this included 2 putative immigrants, M1570 and M1575.

\section{DISCUSSION}

\section{MtDNA diversity compared with other colonies}

The elephant seal colony from KGI had a substantially higher number of mtDNA haplotypes than other contemporary colonies (Table 2). High mtDNA diversity of this moulting colony may result from the presence of immigrants in addition to locally breeding individuals. However, it could also be an effect of the larger sample size for KGI (275 individuals) compared with other colonies studied previously (6-57 individuals). For example, the rarefaction curve for South Georgia shows almost linear growth in the number of haplotypes with increasing sample size (Fig. S1), and we estimated that only 9\% of haplotypes were sampled there, while $53 \%$ of haplotypes were sampled in KGI. Nucleotide diversity in the KGI colony, which is a measure less dependent on the sample size, was within the range of other colonies (Table 2).

Possible immigration to KGI from other breeding colonies in the moulting season is consistent with the increase in abundance of elephant seals in Admiralty Bay after each breeding season (Salwicka \& Rakusa-
Suszczewski 2002). The alternative explanation involves a large number of individuals from KGI skipping the breeding season every year. Southern elephant seals are considered to return to moult to the island on which they breed (Nicholls 1970, Lewis et al. 1996), which would make this second hypothesis more likely. This would imply that either KGI has a high percentage of individuals in reduced reproductive condition, or it has more subadults than reproductive individuals. However, females that haul out on KGI for the breeding season were shown to be heavier than those from other colonies, gain more mass between lactation and moult, and wean heavier pups (Burton et al. 1997, Carlini et al. 1997, 1999). Therefore, a hypothesis that a large number of individuals from KGI are in reduced reproductive condition is unlikely. There is no evidence for recent growth of the KGI population: Salwicka \& RakusaSuszczewski (2002) reported stable population sizes since 1988. Therefore, it is unlikely that subadults in this population outnumber reproductive individuals.

However, there is strong direct evidence that not all individuals of this species show breeding site fidelity during the moulting season. For example, Oosthuizen et al. (2011) documented haulouts of 199 individuals from Iles Crozet (constituting approximately $12 \%$ of the tagged population there) at Marion Island during $15 \mathrm{yr}$, and most of these haulouts occurred in the moulting season. Moreover, at other breeding sites the numbers of elephant seals decline after the breeding season (e.g. Laws 1984, Slip \& Burton 1999). This includes the South Georgia colony (Laws 1984), which constitutes $>99 \%$ of the South Atlantic population and approximately $54 \%$ of the global population (Boyd et al. 1996). Decline after the breeding season implies that a part of the South Georgia's breeding population must moult elsewhere, and other South Atlantic islands, including KGI, are the likely destination.

This is strongly supported by satellite telemetry studies of southern elephant seals. Bornemann et al. (2000) reported that 2 of 9 post-moult adult females fitted with satellite transmitters at KGI hauled out on South Georgia during a breeding season. Similarly, Tosh et al. (2009) reported that an adult male fitted with a satellite transmitter at KGI hauled out on South Georgia at the beginning of a breeding season. McConnell et al. (1992) reported that one of 3 postbreeding females fitted with satellite transmitters at South Georgia was sighted in the following moulting season on KGI. This provides strong evidence that some individuals breeding on South Georgia migrate to KGI for moulting. There is also evidence for move- 
ments of elephant seals between KGI and the adjacent Elephant Island (McConnell et al. 1992, Bornemann et al. 2000, Muelbert et al. 2004). Consistently, our study showed that $79 \%$ of individuals sampled in KGI had mtDNA haplotypes that have been detected earlier in other South Atlantic colonies on South Georgia, the Falklands and Elephant Island. Taken together, these data support the hypothesis that the moulting colony at KGI, besides individuals breeding at this site, also includes individuals from other South Atlantic breeding colonies.

While haplotype sharing between KGI and other South Atlantic breeding colonies was extensive, we detected only 2 haplotypes (in 4 adult individuals) that have been detected earlier in the south Indian Ocean colonies, and no individuals with haplotypes characteristic for the south Pacific Ocean. This is consistent with earlier studies showing limited migration between breeding sites from these 3 parts of the Antarctic (Slade et al. 1998, Hoelzel et al. 2001). In a study on movements of 85 elephant seals from 4 islands in 3 oceans, Biuw et al. (2007) found extensive overlap between the ranges of individuals from the 2 South Atlantic colonies (South Georgia and Livingston Island), but much less overlap between individuals from colonies located in different oceans (see Fig. 1 therein). These authors suggested that demographic distinctiveness of the populations from different sectors of the Southern Ocean may be a consequence of differing oceanographic conditions and resulting environmental variability. Consistently, the pattern of haplotype sharing between colonies observed in our study suggests extensive movements of individuals between South Atlantic colonies and limited movements between colonies from different oceans.

\section{Genetic diversity and population structure at microsatellite loci}

Heterozygosity at microsatellite loci was in the lower range of values reported from the breeding colonies of the southern elephant seals (Table 2), consistent with the small census size of this colony (525 \pm 62 individuals in the moulting season; Salwicka \& Rakusa-Suszczewski 2002) as compared with other colonies considered (e.g. South Georgia: approx. 400000 ind., Boyd et al. 1996; Peninsula Valdes: approx. 40000 ind., Lewis et al. 1996). The heterozygosity deficit observed in the KGI colony could be explained by a combined effect of sampling bias, null alleles and some level of inbreeding. In- breeding has been supported by the kinship analysis, which indicated that most individuals had relatives within the sampled population. Taking into account the putatively mixed composition of the KGI colony, as inferred from the mtDNA haplotype data, the Wahlund effect could have been expected as a likely explanation of the heterozygosity deficit. However, we failed to detect any genetic structure within the population studied.

\section{Kinship in the KGI moulting colony}

Results of kinship analyses indicated that the KGI colony is composed of groups of related individuals, with only few individuals with no relatives identified within the sampled population. This could reflect a true pattern of relatedness in the KGI colony, but could also result from relatively low genetic diversity of southern elephant seals at microsatellite locia possible effect of population bottlenecks due to extensive hunting in the 18th and 19th centuries.

Only one possible mother-offspring pair and 3 father-offspring pairs were identified (assuming $95 \%$ confidence levels). These low numbers are expected for a population sampled outside of the breeding season. However, we identified a large number of groups related at the half-sibling level. Within most of these groups, individuals had more than one mtDNA haplotype, implying that these groups consisted of either paternal half-siblings or more distant relatives. A large number of paternal half-siblings may be expected in this highly polygynous species. A genetic parentage analysis for the southern elephant seal colony on Sea Lion Island (Falklands), showed that only $28 \%$ of males achieved paternities, $90 \%$ of pups were fathered by harem holders, with up to 32 pups assigned to one harem holder in one breeding season (Fabiani et al. 2004). Therefore, our result is consistent with the species mating system.

\section{Population genetic structure and immigration}

We detected no population genetic structure within the KGI colony, while the presence of structure would be expected if groups of individuals from several different, genetically distinct breeding colonies used KGI as a moulting site. Only 2 individuals were identified as immigrants. However, in the absence of microsatellite data from other colonies for a direct comparison, the power to detect immigrants was low. 
For the 2 individuals identified as immigrants, no close kin were detected in the study population, suggesting that they could have arrived in the KGI only for moulting. Immigrants that reproduced on KGI would be more difficult to detect due to their genetic similarity to other individuals from the colony (i.e. their offspring).

Lack of population structure and a low number of immigrants detected suggests that most individuals moulting at KGI are of local origin. However, effective population size estimates for the KGI population are inconsistent with its genetic isolation. The LDbased estimate for the season 2005/2006 (501 individuals) was close to the census estimate of $525 \pm$ 62 individuals calculated as a mean from 12 moulting seasons (Salwicka \& Rakusa-Suszczewski 2002). However, estimates made separately for adults and pups, when combined, gave only 209 individuals. Similarly, the effective size of 771 individuals estimated for all the individuals sampled in different years was inconsistent with the combined estimate for adults and pups (480 individuals). The fact that these estimates do not sum up, and are much higher than expected for a highly polygynous species (which should have a low $N_{\mathrm{e}} / N$ ratio), suggests that the studied moulting colony is composed of individuals from different breeding populations. This conclusion is additionally supported by the female effective population size estimated from mtDNA for the entire sample set (3571 individuals). Such a high estimate can be reliable only when we assume immigration of individuals from other breeding colonies. The higher estimate of the effective population size based on mtDNA as compared with nuclear DNA results from the highly polygynous mating system of southern elephant seals, and was also observed in an earlier study of this species (Slade et al. 1998).

Estimates of effective population size potentially reflect past size, especially in cases of recently reduced populations. However, we found no evidence for a population bottleneck during the last few generations. This is consistent with the census data showing that the South Atlantic population of southern elephant seals has remained stable since 1950 (Boyd et al. 1996, McMahon et al. 2005), in contrast with the South Pacific and South Indian Ocean populations, where declines have been observed (McMahon et al. 2005). Long-term population decline due to intensive hunting in the 18th and 19th centuries could have resulted in the overall decline in genetic diversity of southern elephant seals, but this decline is too distant in time to be detectable in the bottleneck test applied here. However, this past bottleneck could have contributed to the contrasting patters of mitochondrial and nuclear DNA variation (see Fay \& Wu 1999).

While the hypothesis about immigration to KGI from other breeding colonies in the moulting season is supported by mtDNA data, high estimates of $N_{\mathrm{e}}$, and the conclusions from demographic analyses by Salwicka \& Rakusa-Suszczewski (2002), it is inconsistent with the genetic homogeneity of the KGI colony at microsatellite loci. However, as discussed above, detectability of immigrants may be low in the absence of data from the source populations, especially if the source populations are genetically similar to the KGI. Analysis of genetic differentiation at microsatellite loci between 3 other South Atlantic colonies, Elephant Island, South Georgia and Sea Lion Island (Falklands), showed non-significant pairwise $\mathrm{Rho}_{\mathrm{ST}}$ (an unbiased version of the $\mathrm{R}_{\mathrm{ST}}$ coefficient that accounts for differences in allele sizes) values (Fabiani 2002), suggesting ongoing or recent gene flow among these colonies. KGI shares mtDNA haplotypes with each of the abovementioned colonies, and therefore it is likely to have a similar genetic composition at microsatellite loci as well.

Despite breeding site fidelity, cases of breeding dispersal between colonies, usually within the same sector of the Southern Ocean, have been described for both males and females (Bester 1989, Guinet et al. 1992, Fabiani et al. 2003, Reisinger \& Bester 2010, Oosthuizen et al. 2011; see the Supplement for details). In this highly polygynous species, male dispersal is of particular significance, because a male immigrant that succeeds as a harem holder contributes highly to the gene pool of the colony he joins. For example, Oosthuizen et al. (2011) documented a case where a male from Possession Island (Iles Crozet) controlled harems at Marion Island for 6 consecutive breeding seasons, and during this period 196 females ( $7.6 \%$ of all breeding females) passed through his harems. Such rare cases of successful breeding dispersal act as a homogenising force that strongly reduces genetic differentiation between different colonies.

\section{CONCLUSIONS}

The moulting colony of the southern elephant seal from KGI exhibits high variability of mtDNA haplotypes and a high percentage of haplotypes shared with other elephant seal colonies from the South Atlantic. This pattern, coupled with a 3-fold increase in population numbers between the breeding and 
moulting seasons (Salwicka \& Rakusa-Suszczewski 2002), suggests that this colony is composed of both residents and immigrants. This is consistent with high estimates of the effective population size (based on both mtDNA and microsatellite data) that could not be sustained by a small local population. The lack of genetic structure and low number of immigrants detected based on microsatellite loci may result from the genetic similarity between KGI and other breeding colonies from the South Atlantic, and/or an insufficient sampling scheme. A further study including samples collected during the breeding season from the KGI colony as well as samples from other colonies of southern elephant seals is needed to better understand the patterns of their movements between breeding and moulting sites.

The southern elephant seal is an important model species used to address a number of general questions such as species response to climate change (de Bruyn et al. 2009) and sensitivity of marine top predators to large-scale variability of oceanographic conditions (Biuw et al. 2007). Understanding the patterns of migration and gene flow in this species is essential to adequately address these general questions.

Acknowledgements. We are grateful to M. Korczak and K. J. Chwedorzewska for data on elephant seal spatial distribution in Admiralty Bay, and to A. Tereba for her assistance in the laboratory. We thank R. Van Den Bussche, P. Palsbøll and 3 anonymous reviewers for their helpful comments on the manuscript. This study was partially funded by the Committee for Scientific Research (Polish National Polar Project No. PBZ-KBN-108/P04/2004: Structure, evolution and dynamics of the lithosphere, cryosphere and biosphere in the European sector of the Arctic and in the Antarctic). M.P. was supported by a fellowship from the Foundation for Polish Science.

\section{LITERATURE CITED}

> Allen PJ, Amos W, Pomeroy PP, Twiss SD (1995) Microsatellite variation in grey seals (Halichoerus grypus) shows evidence of genetic differentiation between two British breeding colonies. Mol Ecol 4:653-662

Bandelt HJ, Forster P, Röhl A (1999) Median-joining networks for inferring intraspecific phylogenies. Mol Biol Evol 16:37-48

Bester MN (1989) Movements of southern elephant seals and subantarctic fur seals in relation to Marion Island. Mar Mamm Sci 5:257-265

Biuw M, Boehme L, Guinet C, Hindell M and others (2007) Variations in behavior and condition of a Southern Ocean top predator in relation to in situ oceanographic conditions. Proc Natl Acad Sci USA 104:13705-13710

Bornemann H, Kreyscher M, Ramdohr S, Martin T, Carlini A, Selmann L, Plötz J (2000) Southern elephant seal movements and Antarctic sea ice. Antarct Sci 12:3-15

Boyd IL, Walker TR, Poncet J (1996) Status of southern ele- phant seals at South Georgia. Antarct Sci 8:237-244

Bradshaw CJA, Hindell MA, Sumner MD, Michael KJ (2004) Loyalty pays: potential life history consequences of fidelity to marine foraging regions by southern elephant seals. Anim Behav 68:1349-1360

Brown SDI, Collins RA, Boyer S, Lefort MC, MalumbresOlarte J, Vink CI, Cruickshank RH (2012) SPIDER: An R package for the analysis of species identity and evolution, with particular reference to DNA barcoding. Mol Ecol Resour 12:562-565

Burton HR, Arnbom T, Boyd IL, Bester N, Vergani D, Wilkinson I (1997) Significant differences in weaning mass of southern elephant seals from five sub-Antarctic islands in relation to population declines. In: Battaglia B, Valencia J, Walton DWH (ed) Antarctic communities: species, structure and survival. Cambridge University Press, Cambridge, p 335-338

Carlini AR, Danerig A, Marquez MEI, Soaveg E, Poljak S (1997) Mass transfer from mother to pups and mass recovery by mothers during the post-breeding foraging period in southern elephant seals (Mirounga leonina) at King George Island. Polar Biol 18:305-310

Carlini AR, Marquez MEI, Danerig A, Poljak S (1999) Mass changes during their annual cycle in females of southern elephant seals at King George Island. Polar Biol 21: 234-239

Carrick R, Csordas SE, Ingham SE (1962) Studies on the southern elephant seal, Mirounga leonina (L.) IV. Breeding and development. CSIRO Wildl Res 7:161-197

Chao A, Lee SW (1992) Estimating the number of classes via sample coverage. J Am Stat Assoc 87:210-217

Chapuis MP, Estoup A (2007) Microsatellite null alleles and estimation of population differentiation. Mol Biol Evol 24: 621-631

> Cornuet JM, Luikart G (1996) Description and power analysis of two tests for detecting recent population bottlenecks from allele frequency data. Genetics 144: 2001-2014

Curtis C, Stewart BS, Karl SA (2007) Sexing pinnipeds with ZFX and ZFY loci. J Hered 98:280-285

Davis CS, Gelatt TS, Siniff D, Strobeck C (2002) Dinucleotide microsatellite markers from the Antarctic seals and their use in other pinnipeds. Mol Ecol Notes 2: 203-208

> de Bruyn M, Hall BL, Chauke LF, Baroni C, Koch PL, Hoelzel AR (2009) Rapid response of a marine mammal species to Holocene climate and habitat change. PLoS Genet 5:e1000554

Excoffier L (2004) Patterns of DNA sequence diversity and genetic structure after a range expansion: lessons from the infinite-island model. Mol Ecol 13:853-864

Excoffier L, Lischer HEL (2010) Arlequin suite ver 3.5: a new series of programs to perform population genetics analyses under Linux and Windows. Mol Ecol Resour 10: 564-567

> Excoffier L, Smouse PE, Quattro JM (1992) Analysis of molecular variance inferred from metric distances among DNA haplotypes: application to human mitochondrial DNA restriction data. Genetics 131:479-491

Fabiani A 2002. Molecular ecology of southern elephant seals (Mirounga leonina): mating system and genetics. $\mathrm{PhD}$ thesis, Durham University

Fabiani A, Hoelzel AR, Galimberti F, Muelbert MMC (2003) Long range paternal gene flow in the southern elephant seal. Science 299:676 
Fabiani A, Galimberti F, Sanvito S, Hoelzel AR (2004) Extreme polygyny among southern elephant seals on Sea Lion Island, Falkland Islands. Behav Ecol 15: 961-969

Fabiani A, Galimberti F, Sanvito FS, Hoelzel AR (2006) Relatedness and site fidelity at the southern elephant seal, Mirounga leonina, breeding colony in the Falkland Islands. Anim Behav 72:617-626

Fay JC, Wu CI (1999) A human population bottleneck can account for the discordance between patterns of mitochondrial versus nuclear DNA variation. Mol Biol Evol 16:1003-1005

Fu YX (1997) Statistical tests of neutrality of mutations against population growth, hitchhiking and background selection. Genetics 147:915-925

Guinet C, Jouventin P, Weimerskirch H (1992) Population changes, movements of southern elephant seals on Crozet and Kerguelen archipelagos in the last decades. Polar Biol 12:349-356

Hindell MA, Little GJ (1988) Longevity, fertility and philopatry of two female southern elephant seals (Mirounga leonina) at Macquarie Island. Mar Mamm Sci 4:168-171

> Hoelzel AR, Halley J, O'Brien SJ, Campagna C and others (1993) Elephant seal genetic variation and the use of simulation models to investigate historical population bottlenecks. J Hered 84:443-449

> Hoelzel AR, Le Boeuf BJ, Reiter J, Campagna C (1999) Alpha male paternity in elephant seals. Behav Ecol Sociobiol 46:298-306

> Hoelzel AR, Campagna C, Arnbom T (2001) Genetic and morphometric differentiation between island and mainland southern elephant seal populations. Proc Biol Sci 268:325-332

Hofmeyr GJG (2000) Dispersal and dispersion of southern elephant seals at Marion Island. MS thesis, University of Pretoria

> Jonker FC, Bester MN (1998) Seasonal movements and foraging areas of southern female elephant seals, Mirounga leonina, from Marion Island. Antarct Sci 10:21-30

Kalinowski ST, Taper ML, Marshall TC (2007) Revising how the computer program CERVUS accommodates genotyping error increases success in paternity assignment. Mol Ecol 16:1099-1106

Konovalov DA, Manning C, Henshaw MT (2004) KINGROUP: a program for pedigree relationship reconstruction and kin group assignments using genetic markers. Mol Ecol Notes 4:779-782

Laws RM (1984) Seals. In: Laws RM (ed) Antarctic ecology, Vol 2. Academic Press, London, p 621-715

> Lewis M, Campagna C, Quintana F (1996) Site fidelity and dispersion of southern elephant seals from Patagonia. Mar Mamm Sci 12:138-147

Marshall TC, Slate J, Kruuk LEB, Pemberton JM (1998) Statistical confidence for likelihood-based paternity inference in natural populations. Mol Ecol 7:639-655

McCann TS (1985) Size, status and demography of southern elephant seal (Mirounga leonina) populations. In: Ling JK, Bryden MM (eds) Studies of sea mammals in south latitudes. Proc Symp 52nd ANZAAS Congr Sydney, May 1982. South Australian Museum, Northfield, p 1-17

> McConnell BJ, Chambers C, Fedak MA (1992) Foraging ecology of southern elephant seals in relation to the bathymetry and productivity of the Southern Ocean. Antarct Sci 4:393-398

McMahon CR, Bester MN, Burton HR, Hindell MA, Brad- shaw CJA (2005) Population status, trends and a reexamination of the hypotheses explaining the recent declines of the southern elephant seal Mirounga leonina. Mammal Rev 35:82-100

> Muelbert MMC, Robaldo RB, Martínes PE, Colares EP, Bianchini A, Setzer A (2004) Movement of southern elephant seals (Mirounga leonina L.) from Elephant Is., South Shetlands, Antarctica. Braz Arch Biol Technol 47: 461-467

> Nicholls DG (1970) Dispersal and dispersion in relation to birthsite of the southern elephant seal, Mirounga leonina (L.) of Macquarie Island. Mammalia 34:598-616

Oosthuizen WC, Bester MNN, Tosh CA, Guinet C, Besson D, de Bruyn PJN (2011) Dispersal and dispersion of southern elephant seals in the Kerguelen province, Southern Ocean. Antarct Sci 23:567-577

Ovenden JR, Peel D, Street R, Courtney AJ, Hoyle SD, Peel SL, Podlich H (2007) The genetic effective and adult census size of an Australian population of tiger prawns (Penaeus esculentus). Mol Ecol 16:127-138

Paetkau D, Calvert W, Stirling I, Strobeck C (1995) Microsatellite analysis of population structure in Canadian polar bears. Mol Ecol 4:347-354

Paetkau D, Slade R, Burden M, Estoup A (2004) Genetic assignment methods for the direct, real-time estimation of migration rate: a simulation-based exploration of accuracy and power. Mol Ecol 13:55-65

> Palsbøll PJ, Peery MZ, Berube M (2010) Detecting populations in the 'ambiguous' zone: kinship-based estimation of population structure at low genetic divergence. Mol Ecol Resour 10:797-805

Peakall R, Smouse PE (2006) GENALEX 6: genetic analysis in Excel. Population genetic software for teaching and research. Mol Ecol Notes 6:288-295

Piry S, Luikart G, Cornuet JM (1999) BOTTLENECK: a computer program for detecting recent reductions in the effective population size using allele frequency data. J Hered 90:502-503

Piry S, Alapetite A, Cornuet JM, Paetkau D, Baudouin L, Estoup A (2004) GENECLASS2: a software for genetic assignment and first-generation migrant detection. J Hered 95:536-539

> Pritchard JK, Stephens M, Donnelly P (2000) Inference of population structure using multilocus genotype data. Genetics 155:945-959

Raymond M, Rousset F (1995) GENEPOP (version 1.2): population genetics software for exact tests and ecumenicism. J Hered 86:248-249

Reeves RR, Stewart BS, Clapham PJ, Powell JA (2002) Guide to marine mammals of the world. A. A. Knopf, New York, NY

> Reisinger RR, Bester MN (2010) Long distance breeding dispersal of a southern elephant seal. Polar Biol 33: 1289-1291

> Rogers AR (1995) Genetic evidence for a Pleistocene population explosion. Evolution 49:608-615

Salwicka K, Rakusa-Suszczewski S (2002) Long-term monitoring of Antarctic pinnipeds in Admiralty Bay (South Shetlands, Antarctica). Acta Theriol 47:443-457

> Slade RW, Moritz C, Hoelzel AR, Burton HR (1998) Molecular population genetics of the southern elephant seal Mirounga leonina. Genetics 149:1945-1957

Slip DJ, Burton H (1999) Population status and seasonal haul-out patterns of the southern elephant seal (Mirounga leonina) at Heard Island. Antarct Sci 11:38-47 
Tajima F (1989) The effect of change in population size on DNA polymorphism. Genetics 123:597-601

Tamura K, Peterson D, Peterson N, Stecher G, Nei M, Kumar S (2011) MEGA5: Molecular evolutionary genetics analysis using maximum likelihood, evolutionary distance, and maximum parsimony methods. Mol Biol Evol 28: 2731-2739

Tosh CA, Bornemann H, Ramdohr S, Schröder M and others (2009) Adult male southern elephant seals from King

Editorial responsibility: Per Palsböll,

Groningen, Netherlands
George Island utilize the Weddell Sea. Antarct Sci 21: 113-121

van Oosterhout C, Hutchinson WF, Wills DMP, Shipley P (2004) MICRO-CHECKER: software for identifying and correcting genotyping errors in microsatellite data. Mol Ecol Notes 4:535-538

- Wilkinson IS, van Aarde RJ (1999) Marion Island elephant seals: the paucity-of-males hypothesis tested. Can J Zool $77: 1547-1554$

Submitted: March 22, 2012; Accepted: November 23, 2012 Proofs received from author(s): March 7, 2013 